\title{
Hematidrosis: a hematology challenge
}

\begin{abstract}
Hematidrosis, also known as bloody sweat is an extremely rare condition and is characterised by blood mixed with sweat oozing out skin and mucosa. The exact incidence of this condition is unknown and is still restricted to isolated case reports from across the world. Though its etiopathogenesis remains unclear, is precipitated during conditions of extreme stress, which can be physical, emotional, or psychological. Leonardo Da Vinci reported a soldier who sweated blood before one of the battles and there have been several other observations seen in individuals before execution since ancient times. It is hypothesised that capillary blood vessels feeding the sweat glands rupture, causing exudation of blood on the surface mixed with sweat like material. It is a physician challenge as the patient is often subjected to multiple investigations and seldom has to face social stigma associated with it. This condition is usually self resolving once the stressor is controlled. In this report, we describe the case of a young boy who presented with recurrent bleeding episodes and was diagnosed to be suffering from Hematidrosis and briefly discuss the associated literature.
\end{abstract}

Keywords: hematidrosis, psychological factors, vasculitic disorders, autonomic disturbances, capillary rupture

\author{
Volume 4 Issue 5 - 2017
}

\section{Shirali Agarwal, Sirisha Rani S}

Department of Pediatric Hemato-Oncology, Rainbow Children's Hospital, India

Correspondence: Sirisha Rani, Senior Consultant, Department of Pediatric Hemato-Oncology, Rainbow Children's Hospital, Hyderabad, India, Email lokisiri@gmail.com

Received: April II, 2017 | Published: May 23, 2017
Abbreviations: PT, prothrombin time; APTT, activated partial thromboplastin time; ANA, anti nuclear antibody

\section{Introduction}

Hematidrosis is an extremely rare phenomenon characterized by blood oozing from skin and mucosa. Technically, it refers to bloody sweat where blood is mixed with sweat like material. It is a condition in which the capillary blood vessels feeding the sweat glands rupture, causing exudation of blood. ${ }^{1}$ It usually occurs under conditions of extreme physical or emotional stress and in general clinical symptomatology responds once the triggering factor is corrected. Very few cases of hematidrosis have been reported in world literature. The exact etiopathogenesis remains unknown. We describe here a case of a 7-year-old male child who presented with recurrent episodes of bleeding and was eventually diagnosed to be suffering from hematidrosis.

\section{Case description}

A 7-year-old male child presented with complaints of recurrent episodes of bleeding from Right thumb nail bed since duration of one month. Each bleeding episode lasted for about 10seconds, usually during the day and subsided spontaneously. The consistency of blood was however thinner and it appeared as blood stained fluid. There were no underlying scratches or trauma on the skin. There were no bleeding manifestations from any other site. There was no history of trauma, jaundice or prolonged bleeding during injuries. No history of any drug intake or blood transfusion. There was no significant past history or any family history of bleeding disorders. There was no consanguinity in the family and birth history was normal. He was academically very good in school and no apparent deviation of normal behaviour was noticed by the parents. At the time of examination in our institute there was no active bleeding hence biopsy of the local site could not be done. His general and systemic examinations were completely normal. Local examination of the site did not reveal any skin breach or swelling or injury. Microscopic analysis of the bloodstained fluid done in coordination with referral hospital later during active bleeding (Figure 1) revealed same components as of peripheral blood-RBC, Leucocytes, Platelets including wax \& epithelial cells. The blood group of the collected blood was matching with child's blood group. Complete blood count and routine blood biochemistry was within normal limits. His Bleeding time, Prothrombin time (PT), Activated Partial Thromboplastin time (APTT), Thrombin time were normal. His von-Willebrand factor Antigen level and ristocetin cofactor assay revealed no abnormality. Fibrinogen and Factor XIII levels were normal. Platelets function tests were normal. Anti Nuclear Antibody (ANA) levels was normal, also child did not have any clinical evidence of vasculitis. However, skin biopsy, which is definitive for this condition and should be performed during the time of active bleeding, could not be done as child came to us after bleeding stopped. A diagnosis of hematidrosis was considered and he was subjected to environmental changes and Propranolol was started in the dose of $1 \mathrm{mg} / \mathrm{kg}$ with baseline monitoring of vitals and ECG. However, a definitive stressor could not be found except that he was academically brilliant and always a topper (which can be a stressful factor) and they used to live in hot weather. Change in surroundings, more attention from the parents, counselling about studies along with Propranolol did bring down the number of episodes significantly.

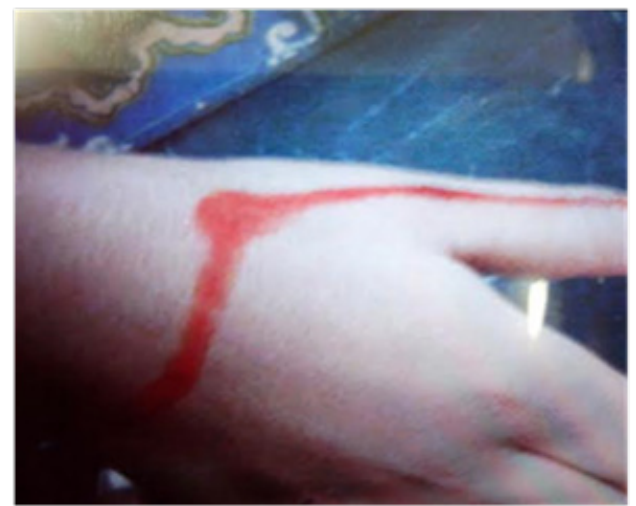

Figure I Characteristic bloody fluid oozing from nail bed. The consistency of blood is thinner. 


\section{Discussion}

Hematidrosis is also known as hematohidrosis and hematidrosis There are several theories for etiopathogenesis of hematidrosis but none of them is conclusive. One hypothesis proposes that extreme stress causes constriction of multiple blood vessels, which are present in a net-like form around the sweat gland. The blood oozes into the sweat glands, which push it along with sweat to the surface, presenting as droplets of blood mixed with sweat. ${ }^{2}$ The extravasated blood has identical cell components as that of peripheral blood. It occurs in extreme physical or emotional stress. Vicarious menstruation, excessive exertion, psychogenic and various unknown causes have thought to be associated, but none proven. Underlying vasculitis disorders or conditions triggering autonomic disturbances needs to be looked at actively. Immediate skin biopsy, during the episode of bleeding is important for definitive diagnosis. Zhang et al. ${ }^{3}$ observed some intradermal bleeding and obstructed capillaries but the sweat glands, hair follicles and sebaceous glands showed no abnormalities. They concluded that distinctive vasculitis can account as a pathological basis for this condition. ${ }^{3}$ Benzidine test can confirm the presence of hemoglobin. Usually blood investigations reveal no abnormality. No definitive management has been identified so far. It is important to identify the stress, which can be physical, or psychological. Counseling along with pharmacotherapy can yield better results. ${ }^{4}$ Drugs like Propranolol have been tried for this condition. It has been suggested that sympathetic nerve activation might be contributory for these events and $\beta$-adrenoceptor antagonists might be an effective treatment for this disorder. ${ }^{5}$ This phenomenon is usually intermittent and self-resolving.

\section{Conclusion}

It is insistent to understand the importance of diagnosing this condition as it is extremely rare to occur. Subjection to multiple investigations and various medications is one of the major problem which is associated with a lot of parental anxiety and can trigger likelihood of being treated as differently by peers and society which can have impact on psychological aspects. It is important to create awareness amongst the clinicians about this condition as it is easily treatable and high index of suspicion will decrease a lot of burden for the ailing patient.

\section{Acknowledgements}

None.

\section{Conflict of interest}

The author declares no conflict of interest.

\section{References}

1. Manonukul J, Wisuthsarewong W, Chantorn R, et al. Hematidrosis: A pathologic process or stigmata. A case report with comprehensive histopathologic and immunoperoxidase studies. Am J Dermatopathol. 2008;30(2):135-139.

2. Jerajani HR, Jaju B, Phiske MM, et al. Hematohidrosis-A rare clinical phenomenon. Indian J Dermatol. 2009;54(3):290-292.

3. Zhang FK, Zheng YL, Liu JH, et al. Clinical and laboratory study of a case of hematidrosis. Zhonghua Xue Ye Xue Za Zhi. 2004;25(3):147-150.

4. Jayaraman AR, Kannan P, Jayanthini V. An interesting case report of hematohidrosis. Indian J Psychol Med. 2017;39(1):83-85.

5. Wang Z, Yu Z, Su J, et al. A case of hematidrosis successfully treated with propranolol. Am J Clin Dermatol. 2010;11(6):440-443. 